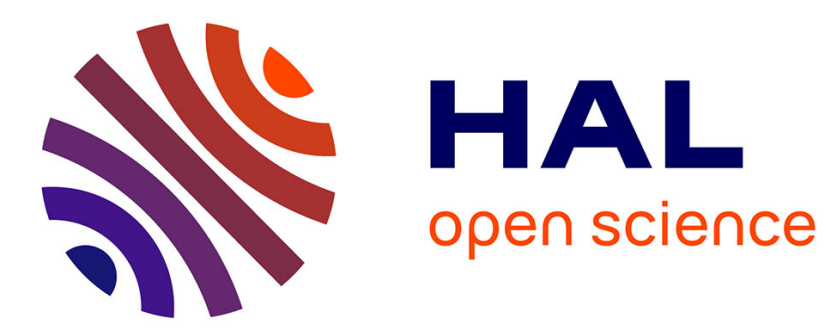

\title{
A simple well-balanced and positive numerical scheme for the shallow-water system
}

Emmanuel Audusse, Christophe Chalons, Philippe Ung

\section{To cite this version:}

Emmanuel Audusse, Christophe Chalons, Philippe Ung. A simple well-balanced and positive numerical scheme for the shallow-water system. Communications in Mathematical Sciences, 2015, 13 (5), pp.1317-1332. 10.4310/CMS.2015.v13.n5.a11 . hal-01083364v2

\section{HAL Id: hal-01083364 \\ https://hal.science/hal-01083364v2}

Submitted on 12 Jan 2015

HAL is a multi-disciplinary open access archive for the deposit and dissemination of scientific research documents, whether they are published or not. The documents may come from teaching and research institutions in France or abroad, or from public or private research centers.
L'archive ouverte pluridisciplinaire HAL, est destinée au dépôt et à la diffusion de documents scientifiques de niveau recherche, publiés ou non, émanant des établissements d'enseignement et de recherche français ou étrangers, des laboratoires publics ou privés. 


\title{
A SIMPLE WELL-BALANCED AND POSITIVE NUMERICAL SCHEME FOR THE SHALLOW-WATER SYSTEM. *
}

\author{
EMMANUEL AUDUSSE ${ }^{\dagger}$, CHRISTOPHE CHALONS $\ddagger$, AND PHILIPPE UNG $\S$
}

\begin{abstract}
This work considers the numerical approximation of the shallow-water equations. In this context, one faces three important issues related to the well-balanced, positivity and entropypreserving properties, as well as the ability to consider vacuum states. We propose a Godunov-type method based on the design of a three-wave Approximate Riemann Solver (ARS) which satisfies the first two properties and a weak form of the last one together. Regarding the entropy, the solver satisfies a discrete non-conservative entropy inequality. From a numerical point of view, we also investigate the validity of a conservative entropy inequality.
\end{abstract}

Key words. shallow-water equations, approximate Riemann solver, finite volume method, positivity preserving, well-balanced scheme.

subject classifications.

\section{Introduction.}

In this work, we look for a numerical scheme for the shallow-water equations given by:

$$
\left\{\begin{array}{l}
\partial_{t} h+\partial_{x}(h u)=0, \\
\partial_{t}(h u)+\partial_{x}\left(h u^{2}+\frac{g h^{2}}{2}\right)=-g h \partial_{x} b(x),
\end{array}\right.
$$

where $b(x)$ is a smooth topography, $g$ refers to the gravitational acceleration, and the water height $h(t, x)$ and the velocity $u(t, x)$ depend on time $t$ and space $x$. We denote $h_{0}$ and $u_{0}$ the initial values respectively for the water height and the velocity. In addition, the associated entropy inequality we will call non-conservative entropy inequality, is written as:

$$
\left\{\begin{array}{l}
\partial_{t} \mathcal{U}(w)+\partial_{x} \mathcal{F}(w) \leqslant-g h u \partial_{x} b \\
\mathcal{U}(w)=\frac{h u^{2}}{2}+\frac{g h^{2}}{2}, \quad \mathcal{F}(w)=\left(\frac{u^{2}}{2}+g h\right) h u
\end{array}\right.
$$

where $w=(h, h u)^{T}$. We also recall the expression of the conservative entropy inequality [6],

$$
\partial_{t} \tilde{\mathcal{U}}(w, b)+\partial_{x} \tilde{\mathcal{F}}(w, b) \leqslant 0,
$$

with the conservative entropy $\tilde{\mathcal{U}}$ and the associated flux $\tilde{\mathcal{F}}$ defined by,

$$
\tilde{\mathcal{U}}(w, b)=\mathcal{U}(w)+g h b \quad \text { and } \quad \tilde{\mathcal{F}}(w, b)=\mathcal{F}(w)+g h u b .
$$

${ }^{\dagger}$ LAGA, UMR CNRS 7539, Université Paris XIII, Sorbonne Paris Cité, F-93430 Villetaneuse, France - INRIA, ANGE Project-Team, Rocquencourt, F-78153 Le Chesnay Cedex, France - CEREMA, ANGE Project-Team, F-60280 Margny-Lès-Compiègne, France - LJLL, UPMC Université Paris VI, ANGE Project-Team, UMR CNRS 7958, F-75005 Paris, France; http://www.math.univ-paris13.fr/ audusse/index.html, (audusse@math.univ-paris13.fr).

${ }^{\ddagger}$ LMV, UMR CNRS 8100, Université de Versailles-Saint-Quentin-en-Yvelines, F-78035 Versailles Cedex, France; http://chalons.perso.math.cnrs.fr/, (christophe.chalons@uvsq.fr).

$\S$ MAPMO, UMR CNRS 7349, Université d'Orléans, F-45067 Orléans Cedex 2, France - INRIA, ANGE Project-Team, Rocquencourt, F-78153 Le Chesnay Cedex, France - CEREMA, ANGE Project-Team, F-60280 Margny-Lès-Compiègne, France - LJLL, UPMC Université Paris VI, ANGE Project-Team, UMR CNRS 7958, F-75005 Paris, France; (philippe.ung@math.cnrs.fr). 
The scheme should preserve the steady states of the lake at rest defined by

$$
\left\{\begin{array}{l}
h_{L}+b_{L}=h_{R}+b_{R} \\
u_{L}=u_{R}=0
\end{array}\right.
$$

and the positivity of water heights for two initial states $(h, h u, b)_{L}$ and $(h, h u, b)_{R}$ separated by a discontinuity, and satisfy a discrete entropy inequality. Furthermore, it should be able to handle vacuum, in particular, the steady state of the wet-dry transition

$$
\left\{\begin{array}{l}
h_{L}+b_{L} \leqslant b_{R}, \quad h_{R}=0 \\
u_{L}=u_{R}=0
\end{array}\right.
$$

or the case of a dry-wet transition,

$$
\left\{\begin{array}{l}
h_{R}+b_{R} \leqslant b_{L}, \quad h_{L}=0, \\
u_{L}=u_{R}=0
\end{array}\right.
$$

There is a huge amount of work about this topic but most of the schemes fail to satisfy these three properties at once. Up to our knowledge, four methods ([18], [6], [11], [4]) are proved to fulfill the three requirements but they are costly in terms of computing runtime and/or based on quite complex algorithms. In this work, we propose a numerical scheme adapted to vacuum that endows the positivity and wellbalanced properties and that is very cheap and simple to implement. We also study the non-conservative entropy (1.3)-(1.4) associated to the scheme and numerically exhibit the decreasing of the discrete conservative entropy (1.5)-(1.6) in a very sharp test case which involves resonance phenomenon. Other numerical experiments are proposed to compare the new method with some positive and well-balanced (but non-entropic) schemes ([12], [13], [2], [5]).

\section{Numerical scheme.}

In the following, we describe a Godunov-type finite volume scheme for (1.1)(1.2)-(1.3)-(1.4). Let us first introduce some notations. We consider a sequence of points $x_{i+1 / 2}$ such that

$$
x_{i-1 / 2}<x_{i+1 / 2}, \quad \forall i \in \mathbb{Z},
$$

and we define the cells $C_{i}$ and space steps $\Delta x_{i}=\Delta x$, such that

$$
\left.C_{i}=\right] x_{i-1 / 2}, x_{i+1 / 2}\left[, \quad \Delta x=x_{i+1 / 2}-x_{i-1 / 2} .\right.
$$

In addition, we set $x_{i}=\left(x_{i-1 / 2}+x_{i+1 / 2}\right) / 2$.

We also introduce a time step $\Delta t>0$ that allows to define a sequence of intermediate times $t^{n}$ by

$$
t^{n+1}=t^{n}+\Delta t .
$$

We denote $\forall X \in\left\{h_{0}, u_{0}, b\right\}$,

$$
\left\{\begin{array}{l}
X_{L}=\frac{1}{\Delta x} \int_{-\Delta x}^{0} X(x) d x, \quad X_{R}=\frac{1}{\Delta x} \int_{0}^{\Delta x} X(x) d x \\
X_{i}=\frac{1}{\Delta x} \int_{C_{i}} X(x) d x
\end{array}\right.
$$

Starting from a given piecewise constant approximate solution at time $t^{n}$, we construct the solution at time $t^{n+1}$ in two steps: 
- we build an approximate solution of the Riemann problem at each interface $x_{i+1 / 2}$,

- we obtain the new solution by calculating the average value of the juxtaposition of these solutions in each cell $C_{i}$ at time $t^{n+1}$.

As an approximate Riemann solution associated with initial data

$$
w(0, x)= \begin{cases}w_{L}, & x<0 \\ w_{R}, & x>0\end{cases}
$$

with $w_{L}=\left(h_{L}, h_{L} u_{L}\right)^{T}$ and $w_{R}=\left(h_{R}, h_{R} u_{R}\right)^{T}$, we consider a simple approximate Riemann solver composed by three waves propagating with velocities $\lambda_{L}, \lambda_{0}=0$ and $\lambda_{R}$ as shown on the following figure

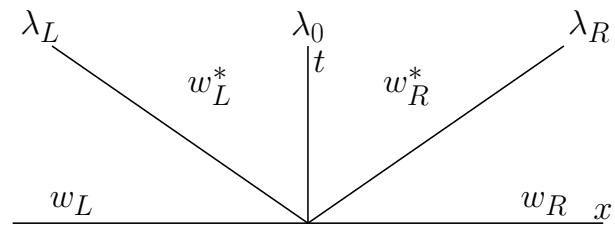

$$
\lambda_{L} \leqslant 0 \leqslant \lambda_{R}
$$

FIG. 2.1. Riemann solver.

We define the CFL condition which ensures that two Riemann problems do not interact

$$
\Delta t<\frac{\Delta x}{2 \max \left(\left|\lambda_{L}\right|, \lambda_{R}\right)},
$$

where the function max is defined in the set of considered Riemann problems. From [15], [12], [13], it is known that such an approximate Riemann solver is consistent in the integral sense with (1.1)-(1.2) provided that the intermediate states satisfy the following consistency relations:

$$
f\left(w_{R}\right)-f\left(w_{L}\right)-s\left(w_{L}, w_{R}, b_{L}, b_{R}\right)=\lambda_{L}\left(w_{L}^{*}-w_{L}\right)+\lambda_{R}\left(w_{R}-w_{R}^{*}\right),
$$

with $f(w)=\left(f^{h}(w), f^{q}(w)\right)^{T}=\left(h u, h u^{2}+g h^{2} / 2\right)^{T}$ and $s\left(w_{L}, w_{R}, b_{L}, b_{R}\right)$ is an approximation of the source term in $(1.1)-(1.2)$, consistent with $(0,-g h \Delta b)^{T}$ since it satisfies:

$$
\lim _{\substack{w_{L}, w_{R} \rightarrow w \\
\Delta x \rightarrow 0}} \frac{1}{\Delta x} s\left(w_{L}, w_{R}, b_{L}, b_{R}\right)=\left(\begin{array}{c}
0 \\
-g h \partial_{x} b
\end{array}\right),
$$

where we recall that $b_{L}$ and $b_{R}$ depend on $\Delta x$ through (2.1). Then, it is also wellknown that the associated Godunov-type scheme is equivalent to the following update formulas:

$$
\left\{\begin{array}{l}
w_{i}^{n+1}=w_{i}^{n}-\frac{\Delta t^{n}}{\Delta x}\left(F_{i+1 / 2}^{L}-F_{i-1 / 2}^{R}\right), \\
w_{i}^{0}=\frac{1}{\Delta x}\left(\int_{C_{i}} h_{0}(x) d x, \int_{C_{i}}\left(h_{0} u_{0}\right)(x) d x\right)^{T},
\end{array}\right.
$$

with $F^{L}\left(w_{i}, w_{i+1}, b_{i}, b_{i+1}\right)$ and $F^{R}\left(w_{i}, w_{i+1}, b_{i}, b_{i+1}\right)$ the left and right numerical fluxes (see [6]) which will be precised later on. 
2.1. Expression of the solution in the intermediate states. We propose to define the two intermediate states by imposing the consistency relations in the integral sense resulting from the equations (1.1)-(1.2):

$$
\left\{\begin{aligned}
h_{R} u_{R}-h_{L} u_{L}=\lambda_{L} & \left(h_{L}^{*}-h_{L}\right)+\lambda_{R}\left(h_{R}-h_{R}^{*}\right) \\
\left(h_{R} u_{R}^{2}+\frac{g h_{R}^{2}}{2}\right)- & \left(h_{L} u_{L}^{2}+\frac{g h_{L}^{2}}{2}\right)+g \Delta x\left\{h \partial_{x} b\right\} \\
& =\lambda_{L}\left(h_{L}^{*} u_{L}^{*}-h_{L} u_{L}\right)+\lambda_{R}\left(h_{R} u_{R}-h_{R}^{*} u_{R}^{*}\right)
\end{aligned}\right.
$$

where $\left\{h \partial_{x} b\right\}$ stands for a consistent approximation of the source term $h \partial_{x} b$ in the sense of (2.6). In order to close this system, two relations are missing and we suggest to impose

$$
\left\{\begin{array}{l}
h_{L}^{*}+b_{L}=h_{R}^{*}+b_{R} \\
h_{L}^{*} u_{L}^{*}=h_{R}^{*} u_{R}^{*}
\end{array}\right.
$$

These two relations are consistent with the steady states (1.7) of the system (1.1)(1.2).

By solving the equations (2.9) and (2.11), we define the water heights in the intermediate states

$$
\begin{aligned}
& h_{L}^{*}=h_{H L L}+\frac{\lambda_{R}}{\lambda_{R}-\lambda_{L}} \Delta b, \\
& h_{R}^{*}=h_{H L L}+\frac{\lambda_{L}}{\lambda_{R}-\lambda_{L}} \Delta b,
\end{aligned}
$$

where $\Delta b=b_{R}-b_{L}$ and

$$
h_{H L L}=\frac{\lambda_{R} h_{R}-\lambda_{L} h_{L}}{\lambda_{R}-\lambda_{L}}-\frac{1}{\lambda_{R}-\lambda_{L}}\left(h_{R} u_{R}-h_{L} u_{L}\right),
$$

is the intermediate water height associated to the HLL solver ([15], [6]).

Then, from the equations (2.10) and (2.12), we deduce the intermediate discharge $q^{*}$

$$
\begin{gathered}
q^{*}:=h_{L}^{*} u_{L}^{*}=h_{R}^{*} u_{R}^{*}, \\
q^{*}=q_{H L L}-\frac{g}{\lambda_{R}-\lambda_{L}} \Delta x\left\{h \partial_{x} b\right\},
\end{gathered}
$$

with

$$
q_{H L L}=\frac{\lambda_{R} h_{R} u_{R}-\lambda_{L} h_{L} u_{L}}{\lambda_{R}-\lambda_{L}}-\frac{\left(h_{R} u_{R}^{2}+\frac{g h_{R}^{2}}{2}\right)-\left(h_{L} u_{L}^{2}+\frac{g h_{L}^{2}}{2}\right)}{\lambda_{R}-\lambda_{L}}
$$

the intermediate discharge involved in the HLL scheme ([15], [6]).

From (2.13), (2.14) and (2.16), we are able to define the two numerical fluxes $F^{L}$ 
and $F^{R}$ :

$$
\left\{\begin{array}{c}
F_{i+1 / 2}^{L}\left(w_{i}, w_{i+1}, b_{i}, b_{i+1}\right)=F_{H L L}\left(w_{i}, w_{i+1}\right)+\left(\begin{array}{c}
\frac{\lambda_{L} \lambda_{R}\left(b_{i+1}-b_{i}\right)}{\lambda_{R}-\lambda_{L}} \\
-\frac{\lambda_{L} g \Delta x\left\{h \partial_{x} b\right\}}{\lambda_{R}-\lambda_{L}}
\end{array}\right)_{i+1 / 2},(2.1 \\
F_{i+1 / 2}^{R}\left(w_{i}, w_{i+1}, b_{i}, b_{i+1}\right)=F_{H L L}\left(w_{i}, w_{i+1}\right)+\left(\begin{array}{c}
\frac{\lambda_{L} \lambda_{R}\left(b_{i+1}-b_{i}\right)}{\lambda_{R}-\lambda_{L}} \\
-\frac{\lambda_{R} g \Delta x\left\{h \partial_{x} b\right\}}{\lambda_{R}-\lambda_{L}}
\end{array}\right)_{i+1 / 2},(2.1
\end{array}\right.
$$

where $F_{H L L}$ is the HLL flux ([15], [6]),

$$
F_{H L L}\left(w_{L}, w_{R}\right)= \begin{cases}f\left(w_{L}\right), & \text { if } 0<\lambda_{L}, \\ \frac{\lambda_{R} f\left(w_{l}\right)-\lambda_{L} f\left(w_{R}\right)}{\lambda_{R}-\lambda_{L}}+\frac{\lambda_{L} \lambda_{R}}{\lambda_{R}-\lambda_{L}}\left(w_{R}-w_{L}\right), & \text { if } \lambda_{L}<0<\lambda_{R}, \\ f\left(w_{R}\right), & \text { if } \lambda_{R}<0,\end{cases}
$$

and $\left\{h \partial_{x} b\right\}$ stands for a discretization of the source term which will be precised later on, see [12], [13], [10], [4]. One notices that the first components of the fluxes $F_{L}$ and $F_{R}$ are equal which means that the scheme is conservative for the water height. The difference between their second components results to the value $\Delta x \times s$, previously defined as a consistent approximation of the source term.

Observe that at this stage the definitions of $\left\{h \partial_{x} b\right\}, \lambda_{L}$ and $\lambda_{R}$ have to be given, while $w_{L}^{*}$ and $w_{R}^{*}$ are the unknows of the equations. The first one will be related to the well-balanced property and the last two will be treated in the next subsection.

2.2. Positivity. In regard to the expression of the intermediate water heights (2.13) and (2.14), it is not easy to ensure the positivity of these quantities. That is why, we suggest to modify these intermediate values depending on the sign of $\Delta b$. In the case $\Delta b \geqslant 0$, we clearly have

$$
h_{R}^{*} \leqslant h_{H L L} \leqslant h_{L}^{*} .
$$

In order to ensure the positivity of $h_{R}^{*}$, we introduce the following modification

$$
\begin{aligned}
& \tilde{h}_{R}^{*}=\max \left(h_{R}^{*}, 0\right), \\
& \tilde{h}_{L}^{*}=h_{L}^{*}-\frac{\lambda_{R}}{\lambda_{L}}\left(h_{R}^{*}-\tilde{h}_{R}^{*}\right),
\end{aligned}
$$

which satisfies the consistency relation (2.9).

One can check the positivity of the intermediate water heights. Indeed, in the case where $\tilde{h}_{R}^{*}=0$, one obtains

$$
\tilde{h}_{L}^{*}=h_{L}^{*}-\frac{\lambda_{R}}{\lambda_{L}} h_{R}^{*}=h_{H L L}\left(1-\frac{\lambda_{R}}{\lambda_{L}}\right) \geqslant 0,
$$

under the same positiveness conditions on $\lambda_{L}$ and $\lambda_{R}$ of the HLL scheme (see [15], [6]). In practice, the following expression for the intermediate velocities work [6]

$$
\begin{aligned}
& \lambda_{L}=\min _{w=w_{L}, w_{R}}(u-\sqrt{g h}, 0), \\
& \lambda_{R}=\max _{w=w_{L}, w_{R}}(u+\sqrt{g h}, 0) .
\end{aligned}
$$


In the following, we will exclusively work with the quantities $\lambda_{R} \tilde{h}_{R}^{*}$ and $\lambda_{L} \tilde{h}_{L}^{*}$,

$$
\begin{aligned}
& \lambda_{R} \tilde{h}_{R}^{*}=\lambda_{R} \max \left(h_{R}^{*}, 0\right), \\
& \lambda_{L} \tilde{h}_{L}^{*}=\lambda_{L} h_{L}^{*}-\lambda_{R}\left(h_{R}^{*}-\tilde{h}_{R}^{*}\right),
\end{aligned}
$$

which will allow us to handle wave velocities equal to zero; in the case $\lambda_{L}=0$, there is no need to define a threshold value.

The case $\Delta b<0$ can be treated by applying the same method and we obtain

$$
\begin{aligned}
& \lambda_{L} \tilde{h}_{L}^{*}=\lambda_{L} \max \left(h_{L}^{*}, 0\right), \\
& \lambda_{R} \tilde{h}_{R}^{*}=\lambda_{R} h_{R}^{*}-\lambda_{L}\left(h_{L}^{*}-\tilde{h}_{L}^{*}\right) .
\end{aligned}
$$

In spite of these modifications of the intermediate water heights, it is important to precise that the expression of the discharge $q^{*}$ is still unchanged.

By modifying the expression of the intermediate water heights, it seems obvious to modify the numerical fluxes (2.17) in respect to the new expression of the intermediate state (2.18) and (2.19).

From now on and as motivated just above, $\lambda_{L}$ and $\lambda_{R}$ refer to the values that ensure the stability properties of the classical HLL scheme (positivity of the water heights but also validity of an entropy inequality as we will need later on).

REMARK 2.1. The intermediate velocities $u_{L}^{*}$ and $u_{R}^{*}$ are never used in practice, in the sense that they are always multiplied by $h$ in the code. In order to treat vacuum states, calculating the values of $u_{L}$ and $u_{R}$ requires to impose a threshold on the water height. Namely, been given $\varepsilon \ll 1$ (in practice, we have taken $\varepsilon=10^{-12}$ ), we set $u_{L}=0$ and $h_{L}=0\left(\right.$ resp. $u_{R}=0$ and $\left.h_{R}=0\right)$ whenever $h_{L}<\varepsilon\left(\right.$ resp. $\left.h_{R}<\varepsilon\right)$.

2.3. Well-balanced property. We introduce the following natural discretization of the source term

$$
\left\{h \partial_{x} b\right\}=\frac{h_{L}+h_{R}}{2 \Delta x} \Delta b,
$$

which preserves the steady states of the lake at rest (1.7).

Indeed, we easily observe that with this definition, one gets

$$
h_{L}^{*}=h_{L}, h_{R}^{*}=h_{R}, q^{*}=0
$$

By modifying this discrete source term as follows

$$
\left\{h \partial_{x} b\right\}= \begin{cases}\frac{h_{L}+h_{R}}{2 \Delta x} \min \left(h_{L}, \Delta b\right), & \text { if } \Delta b \geqslant 0, \\ \frac{h_{L}+h_{R}}{2 \Delta x} \max \left(-h_{R}, \Delta b\right), & \text { if } \Delta b<0,\end{cases}
$$

we can also preserve the lake at rest in the case of a wet-dry transition (1.8) or the case of a dry-wet transition (1.9).

The proof relies again on obvious calculations. 
2.4. About entropy. The entropy-preserving property is an open issue for the proposed scheme since we are not able to prove at present that it satisfies a discrete version of the conservative entropy inequality. Nevertheless, we numerically show that this property is satisfied for a specific test case involving resonance phenomenon (3.5). Actually, the proposed scheme satisfies a discrete version of the non-conservative entropy inequality (1.3)-(1.4) provided that the following conditions on the intermediate states is fulfilled for any $w_{L}, w_{R}, b_{L}, b_{R}$ (see [12]),

$$
\begin{aligned}
& \mathcal{F}\left(w_{R}\right)-\mathcal{F}\left(w_{L}\right)-\sigma\left(w_{L}, w_{R}, b_{L}, b_{R}\right) \leqslant \\
& \lambda_{L}\left(\mathcal{U}\left(w_{L}^{*}\right)-\mathcal{U}\left(w_{L}\right)\right)+\lambda_{R}\left(\mathcal{U}\left(w_{R}\right)-\mathcal{U}\left(w_{R}^{*}\right)\right) .
\end{aligned}
$$

In the aim to be consistent with the shallow-water system, it is necessary to ensure that $\sigma$ is consistent with the term $-g h u \partial_{x} b$ and so we have to satisfy

$$
\lim _{\substack{w_{L}, w_{R} \rightarrow w \\ \Delta x \rightarrow 0}} \frac{1}{\Delta x} \sigma\left(w_{L}, w_{R}, b_{L}, b_{R}\right)=-g h u \partial_{x} b .
$$

We refer again to [15], [12], [13] for more details. In our case, we can express $\sigma$ such as

$$
\sigma\left(w_{L}, w_{R}, b_{L}, b_{R}\right)=-g \frac{h_{L}+h_{R}}{2} \frac{q_{H L L}}{h_{H L L}} \bar{\Delta} b+(\Delta x)^{2} \varepsilon(\Delta x),
$$

where $(\Delta x)^{2} \varepsilon(\Delta x)$ refers to function with the property

$$
\lim _{\Delta x \rightarrow 0} \varepsilon(\Delta x)=0
$$

and

$$
\bar{\Delta} b= \begin{cases}\min \left(h_{L}, \Delta b\right), & \text { if } \Delta b \geqslant 0, \\ \max \left(-h_{R}, \Delta b\right), & \text { if } \Delta b<0 .\end{cases}
$$

The corresponding discrete inequality with the formula (2.29) is clearly consistent with (1.3), at least if $h_{L}>0$ and $h_{R}>0$ since $\overline{\Delta b}=\Delta b$ under these assumptions when $\Delta x \rightarrow 0$.

In the following we propose to examine the behaviour of the solver concerning the non-conservative and conservative entropies, especially for the test case considering non-unique solution to the Riemann problem proposed in [1].

REMARK 2.2. When $\Delta x \rightarrow 0, h_{L}^{*} \rightarrow h_{H L L}, h_{R}^{*} \rightarrow h_{H L L}$ so that (2.18)-(2.19) are never used in this asymptotic regime.

REMARK 2.3. Unlike [4], the proof of the positivity and non-conservative entropy properties are obtained for $\lambda_{L}$ and $\lambda_{R}$ defined exactly as in the HLL scheme, and are not defined asymptotically large according to specific behaviours like $-\lambda_{L} / \lambda_{R} \gg 1$ or $-\lambda_{R} / \lambda_{L} \gg 1$.

\section{Numerical results.}

We are interested in the behaviour of our scheme for different test cases: a propagation of perturbations around equilibrium state [16], [9], a flow over a bump in two regimes (fluvial and transcritical without and with shock), the Thacker test case for 
the wet-dry transition, wet-dry fronts in a nonflat basin [14], [9] and a test for the non-uniqueness of the solution [1].

In what follows, we first show the numerical results provided by the new scheme we propose. For some of them, we also compare the L1-errors with the ones given by a classical HLL scheme coupled with a centered discretization of the source term, the hydrostatic reconstruction [2], the hydrostatic upwind scheme [5], and the scheme proposed by Gallice [12], [13].

3.1. Propagation of perturbations. This test case has been proposed by LeVeque in [16] and reused by Castro et al. in [9]. We perturbed a steady state solution by a pulse that splits into two opposite waves over a continuous bed. The aim is to test the behaviour of the scheme when it is submitted to a rapidly varying flow around the state of the lake at rest. The domain is reduced to the interval $[0,2]$. We work with outflow boundary conditions and the bottom topography is defined by

$$
b(x)= \begin{cases}2+0.25(\cos (10 \pi(x-0.5))+1), & \text { if } 1.4<x<1.6 \\ 2, & \text { otherwise }\end{cases}
$$

The initial datas are $q(0, x)=0$, and

$$
h(0, x)= \begin{cases}3-b(x)+\Delta h, & \text { if } 1.1<x<1.2 \\ 3-b(x), & \text { otherwise }\end{cases}
$$

where $\Delta h=0.001$ is the height of the perturbation. The CFL parameter is set to 0.9. The final time is fixed at $t=0.2$ and $\Delta x=1 / 40$. A reference solution is obtained with the hydrostatic reconstruction applied to the HLL flux with a mesh of 20000 cells.

On Figure 3.3, we isolate the results obtained with a centered scheme that returns a unreasonable solution. The other schemes are well-balanced for the steady state of the lake at rest and the results they return do not present large numerical error as seen before on Figure 3.3. The results in Figure 3.1 and 3.2 show that the scheme introduced in this paper - called simple solver on the figures - is the most accurate.
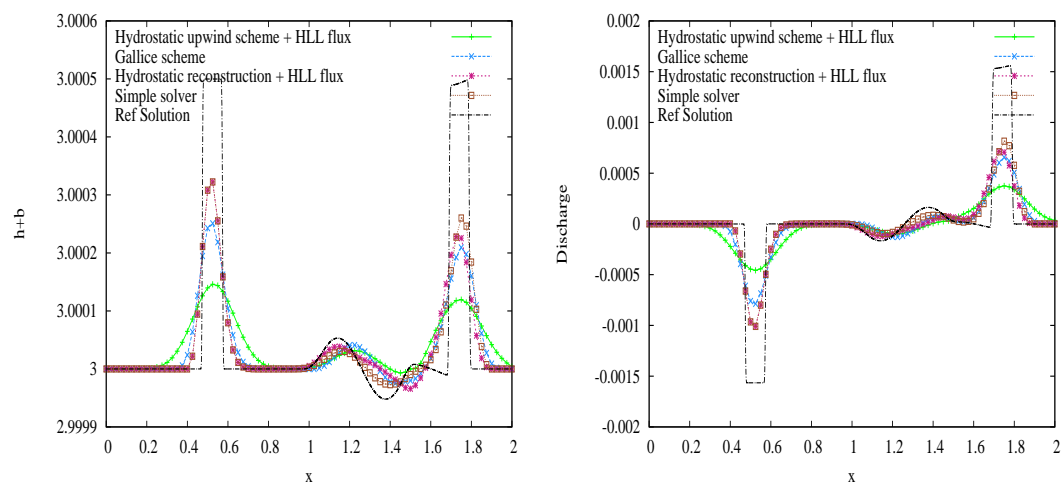

FIG. 3.1. Propagation of perturbations: Comparison of free surfaces $h+b$ (left) and discharges $q$ (right) for different schemes.

3.2. Steady flow over a bump. The aim of these test cases is to bring out the behaviour of the scheme for steady states it has basically not the function to 

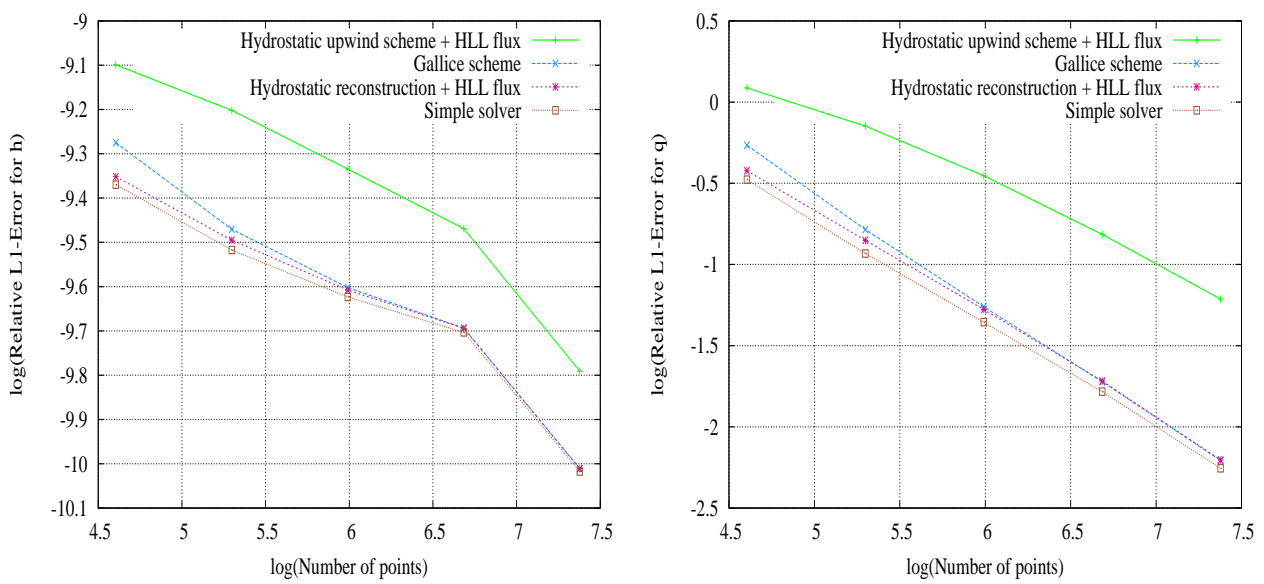

Fig. 3.2. Propagation of perturbations: Comparison of orders of error for the water height $h$ (left) and the discharge $q$ (right) for different schemes.
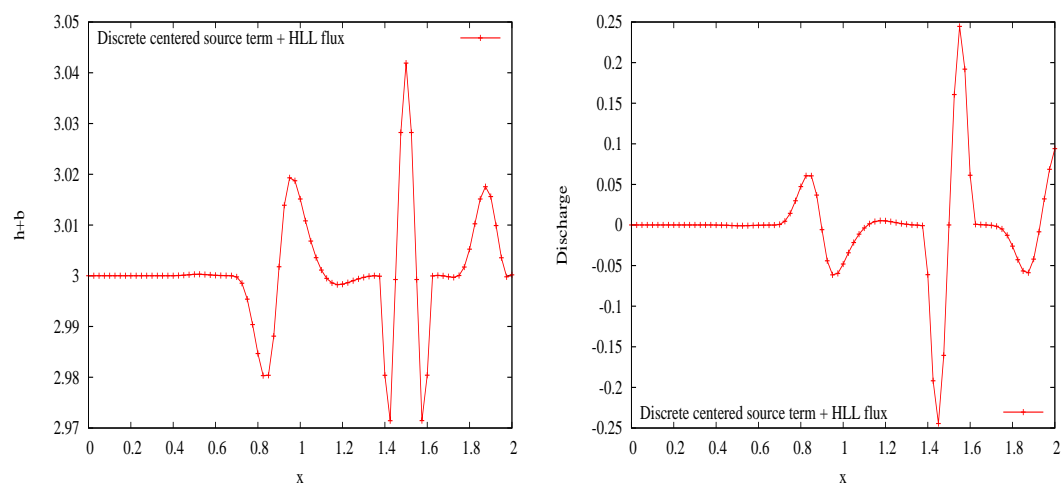

FIG. 3.3. Propagation of perturbations: free surface $h+b$ (left) and discharge $q$ (right) resulting from the centered scheme.

preserve. The steady states are governed by the following equations

$$
h u=K_{1} \quad \text { and } \quad \frac{u^{2}}{2}+g(h+b)=K_{2} .
$$

where $K_{1}$ and $K_{2}$ are two constants. In the following, we deal with the equations (3.3) in the fluvial and transcritical regimes without shock, and the transcritical flow with shock is treated using datas from [9].

3.2.1. Fluvial regime. In this test case, we set $K_{1}=1$ and $K_{2}=25$.

The domain is $[-2 ; 2]$ and the bottom topography is defined by

$$
b(x)= \begin{cases}\frac{\cos (10 \pi(x+1))+1}{4}, & \text { if }-0.1<x \leqslant 0.1, \\ 0, & \text { elsewhere }\end{cases}
$$

The CFL parameter is equal to 0.5 .

The error curves (Fig. 3.5) show that the proposed scheme gives a better approximation of the exact solution than other existing schemes with a gain of several orders. 

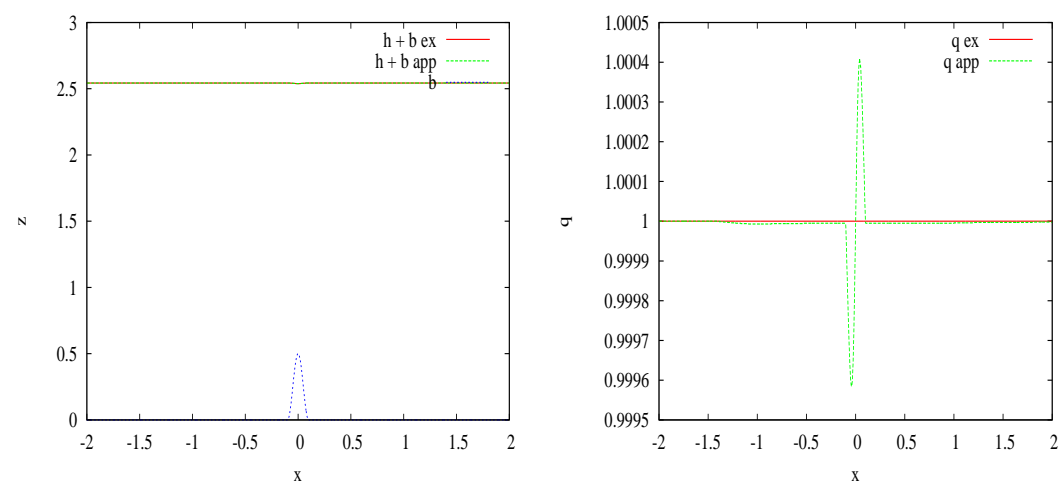

FIG. 3.4. Fluvial flow: Comparison of free surfaces $h+b$ (left) and discharges $q$ (right) resulting from the simple scheme (with suffix app) and the exact solution (with suffix ex) for a bottom topography $b$.
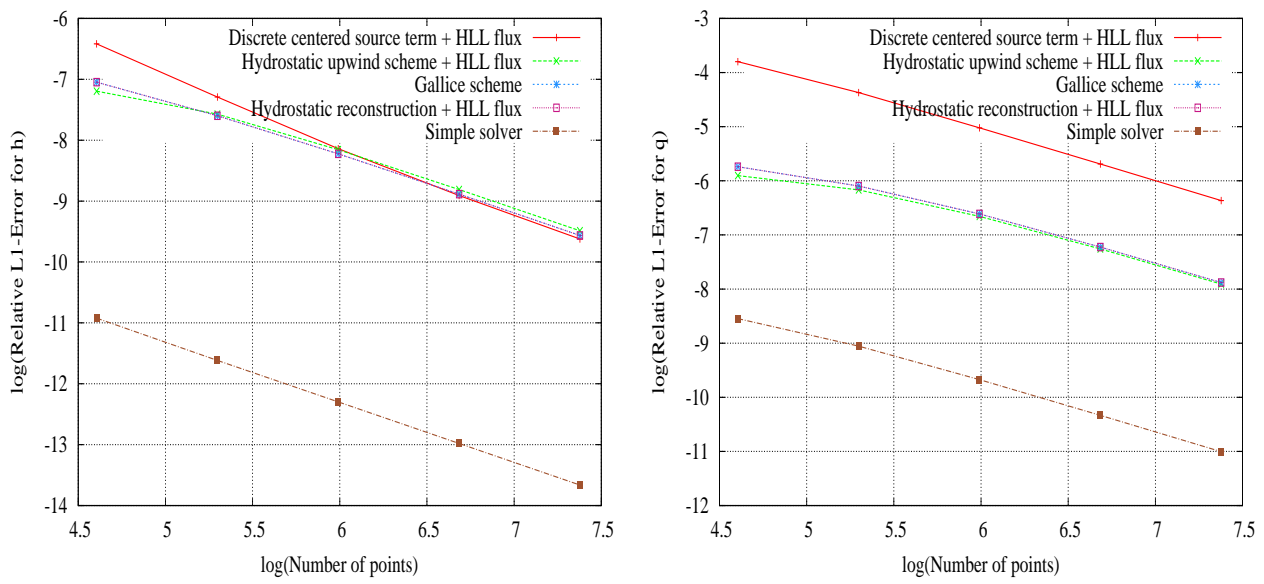

Fig. 3.5. Fluvial flow: Comparison of orders of error for the water height $h$ (left) and the discharge $q$ (right) for different schemes.

3.2.2. Transcritical flow over a bump without shock. This test case sets $K_{1}=3$ and $K_{2}=\frac{3}{2}\left(K_{1} g\right)^{2 / 3}+\frac{1}{2} g$ into the steady state equations (3.3) and the bottom topography is defined by (3.4). The CFL parameter is set to 0.5 .

The observations for the fluvial case also work for the transcritical one but the gain associated to the simple scheme is not as important as the previous one (Fig. 3.7). The reason is probably because we impose opposite signs to the wave velocities and by this way, we add diffusion to the torrential part. Nevertheless, this scheme gives the best approximation.

3.2.3. Transcritical flow over a bump with shock. This test has been proposed by Castro et al. [9]. The domain is the interval [0,25]. The bottom topography is given by

$$
b(x)= \begin{cases}3-0.05(x-10)^{2}, & \text { if } 8<x<12 \\ 2.8, & \text { otherwise }\end{cases}
$$



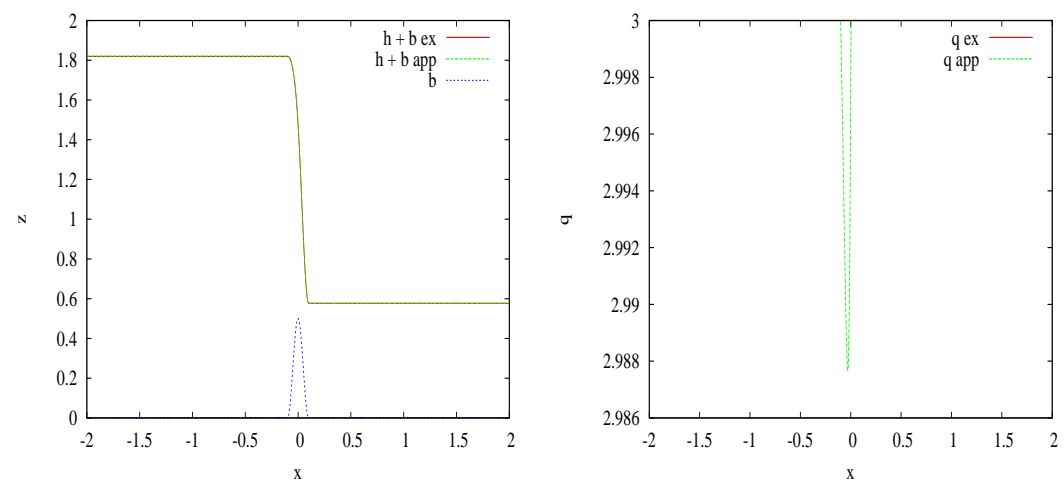

FIG. 3.6. Transcritical flow without shock: Comparison of free surfaces $h+b$ (left) and discharges $q$ (right) resulting from the simple scheme (with suffix app) and the exact solution (with suffix ex) for a bottom topography $b$.
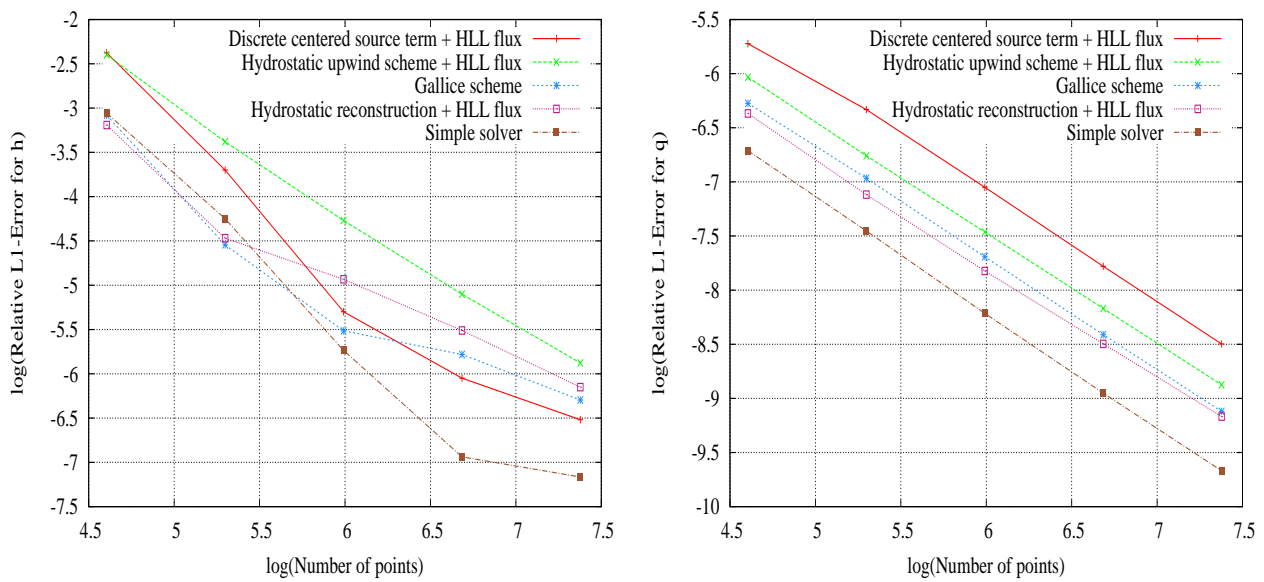

FiG. 3.7. Transcritical flow without shock: Comparison of orders of error for the water height $h$ (left) and the discharge $q$ (right) for different schemes.

The initial state is defined by $h(0, x)=3.13-b(x), q(0, x)=0.18$ and the boundary conditions are $q(t, 0)=0.18$ and $h(t, 25)=0.33$. The final time is set to $t=200$. We work with a CFL parameter equal to 0.9. This test case is focused on the way the scheme handles a stationary shock.

The simple scheme manages to capture the shock as shown in Figure 3.8. The errors on Figure 3.9 reveal that this scheme is again the most accurate compared to other ones.

3.3. Thacker test case for wet-dry transitions. The bottom topography is defined by $\quad b(x)=\frac{x^{2}}{2}+\frac{3}{2} \quad$ for $-2 \leqslant x \leqslant 2$. The explicit periodic solution presents a plane free surface and oscillates between the points

$$
x_{1}(t)=-\frac{1}{2} \cos (\sqrt{g} t)-1 \quad \text { and } \quad x_{2}(t)=-\frac{1}{2} \cos (\sqrt{g} t)+1 .
$$

Here, the pulsation is $\omega=\sqrt{g}$. 

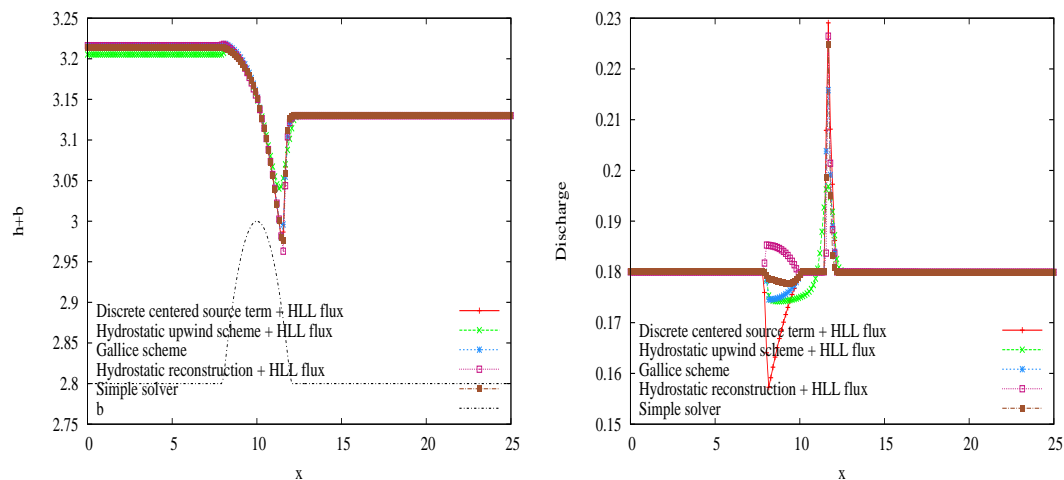

FIG. 3.8. Transcritical flow with shock: Comparison of free surfaces $h+b$ (left) and discharges $q$ (right) for different schemes.
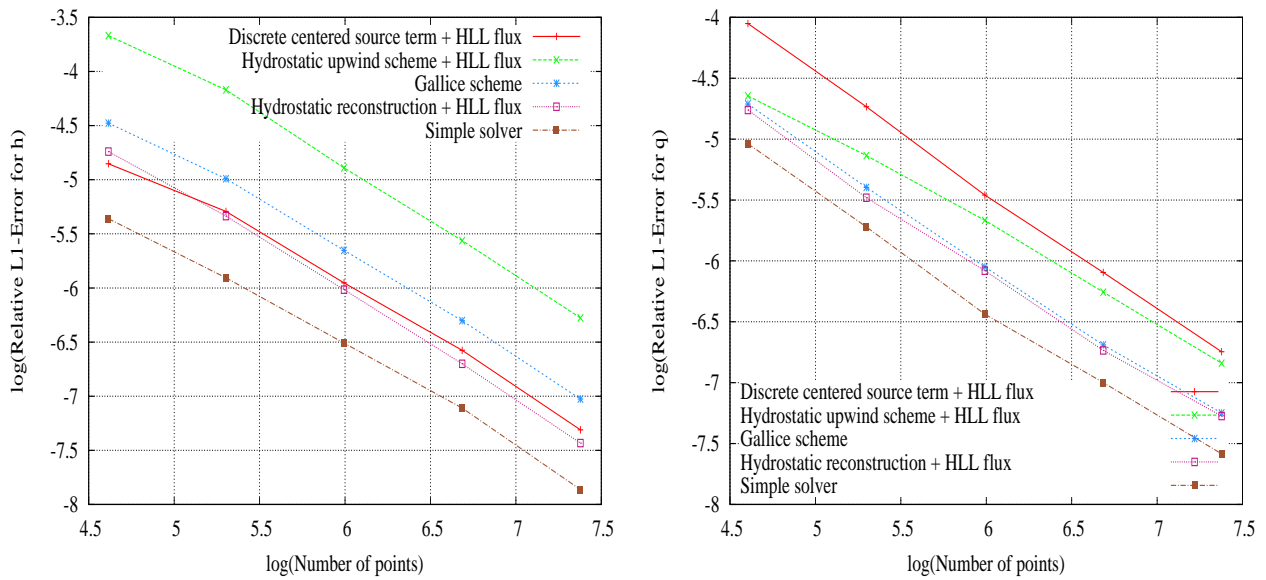

FIG. 3.9. Transcritical flow with shock: Comparison of orders of error for the water height $h$ (left) and the discharge $q$ (right) for different schemes.

The initial conditions are given by $u(0, x)=0$ and

$$
h(0, x)= \begin{cases}-\frac{1}{2}\left(\left(x+\frac{1}{2}\right)^{2}-1\right), & \text { if } x_{1}(0)<x<x_{2}(0), \\ 0, & \text { else } .\end{cases}
$$

The water height and the velocity are expressed as follows

$$
\begin{gathered}
h(t, x)= \begin{cases}-\frac{1}{2}\left(\left(x+\frac{1}{2} \cos (\sqrt{g} t)\right)^{2}-1\right), & \text { if } x_{1}(t)<x<x_{2}(t), \\
0, & \text { else },\end{cases} \\
u(t, x)= \begin{cases}-\frac{\sqrt{g}}{2} \sin (\sqrt{g} t), & \text { if } x_{1}(t)<x<x_{2}(t), \\
0, & \text { else. }\end{cases}
\end{gathered}
$$

We take a value of 0.5 for the CFL parameter. 
This test case shows the capacity of the scheme to handle wet-dry transition. We recall that every considered scheme preserves the non negativity of the water height. We can wisely precise that in this test case, the discharges are very low which can explain the big values of the relative errors one can observe for coarse mesh (Fig. 3.11). In this case, only the hydrostatic reconstruction returns a non accurate solution.
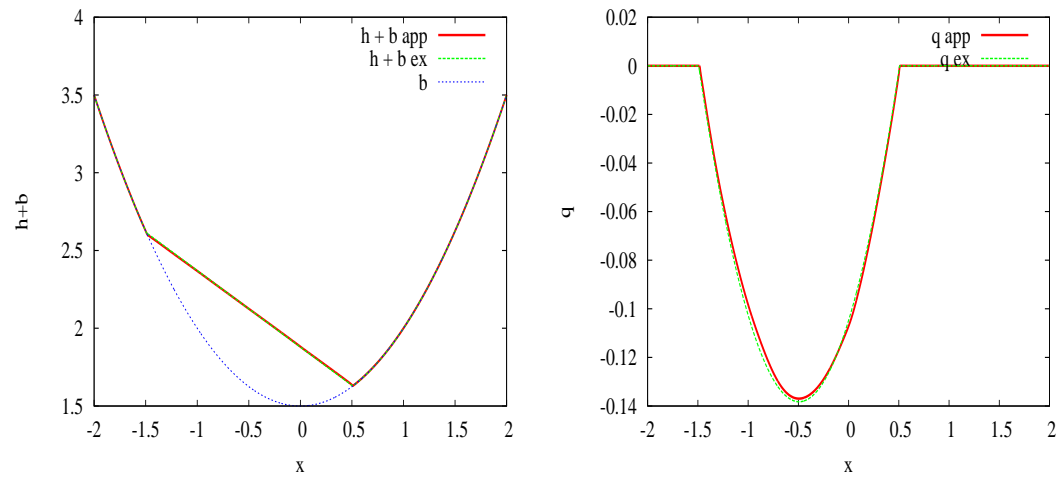

FIG. 3.10. Thacker test case: Comparison of free surfaces $h+b$ (left) and discharges $q$ (right) resulting from the simple scheme (with suffix app) and the exact solution (with suffix ex) for a bottom topography $b$ at time $T=16$.
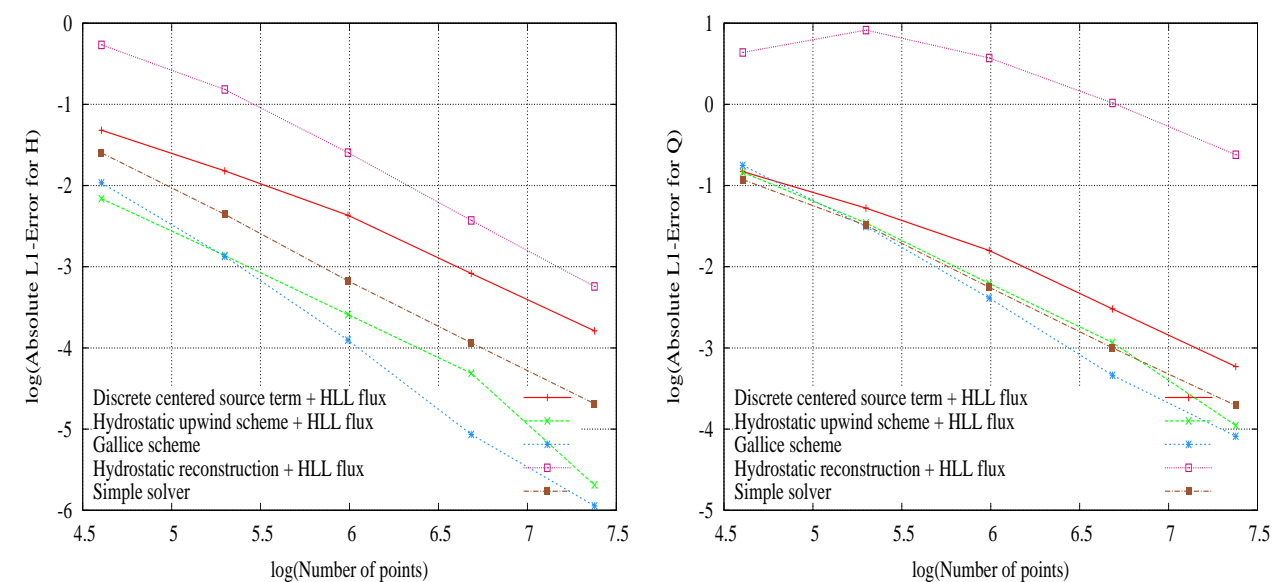

FIG. 3.11. Thacker test case: Comparison of orders of error for the water height $h$ (left) and the discharge $q$ (right) for different schemes at time $T=16$.

3.4. Wet-dry fronts in a nonflat basin. We present another test case for the treatment of the wet-dry transition but in this case, we have a non smooth bottom topography. This problem proposed by Gallouët et al. [14] and also resumed by Castro et al. [9] consists on creating a dry bed in the middle of two rarefaction waves propagating in opposite directions. We work in the space interval $[0,25]$ and the bottom topography is represented by the function

$$
b(x)= \begin{cases}7, & \text { if } 25 / 3<x<25 / 2, \\ 6, & \text { otherwise }\end{cases}
$$


The initial conditions are

$$
q(0, x)= \begin{cases}-300, & \text { if } 50 / 3 \leqslant x \\ 300, & \text { if } 50 / 3>x\end{cases}
$$

and $h(0, x)=16-b(x)$. The CFL parameter is set to 0.9 . A reference solution is computed using the Gallice scheme with a mesh of 20480 points. In this particular case, the difference between the results does not clearly appear (Figure 3.13). It is important to notice that this test case does not present hypothesis satisfying the theoretical properties of our scheme in the sense that it deals with a non smooth topography. In spite of this criterion, the scheme returns a reasonable solution.
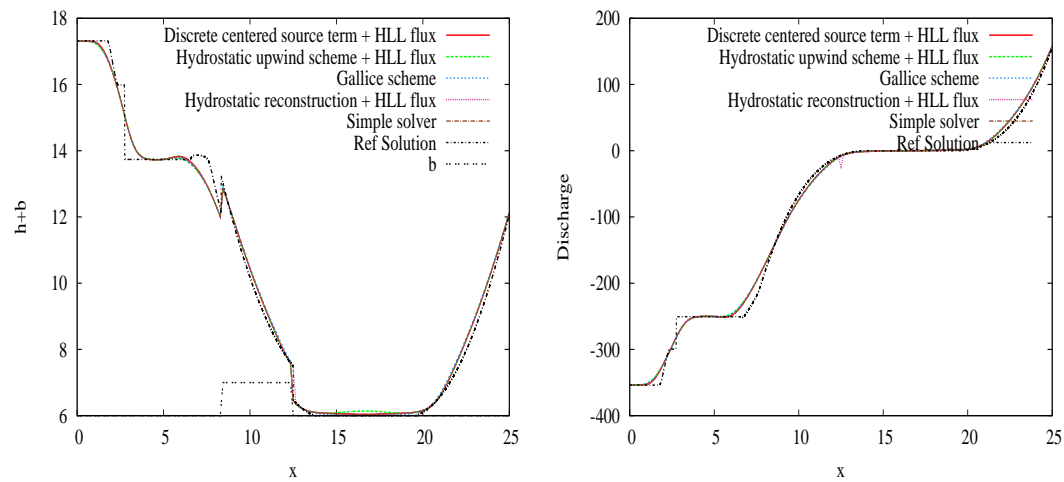

FIG. 3.12. Wet-dry fronts in a nonflat basin: Comparison of free surfaces $h+b$ (left) and discharges $q$ (right) for different schemes.
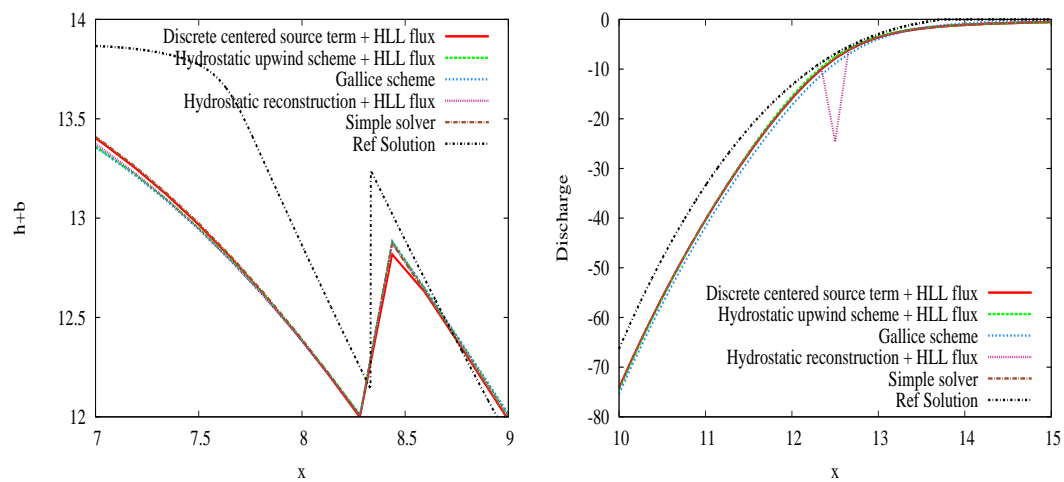

FIG. 3.13. Wet-dry fronts in a nonflat basin: Comparison of free surfaces $h+b$ (left) and discharges $q$ (right) for different schemes.

3.5. Non-unique solution to the Riemann problem. This numerical test case is presented in [1]. We use the same datas presented in the previously mentioned paper which are

$$
(b, h, u)= \begin{cases}(1.5,1.3,-2), & \text { if } x \leqslant 0.5 \\ (1.1,0.1,-2), & \text { if } x>0.5\end{cases}
$$

with $x \in[0,1]$. We set the gravitational acceleration $g$ to 2 . The test problem has non-unique solution. The CFL parameter is set to 0.9 . 
The numerical solutions are separated into two groups which correspond to the two different solutions of the problem. Every considered scheme relatively return the same solution which is one of the previous two solutions whereas the simple scheme returns the second one. This last solution is quite similar to the one obtained with the relaxation solver [6], [9] and the kinetic scheme proposed in [18] that we have especially introduced for this test case and called SVK in the Fig. 3.14 and 3.15, which are both entropic. Nevertheless, these last three solutions are different from the exact one, graphically presented in [1]. As mentioned in this previous article, the reason of this difference is the lack of complete theory for non-conservative system.
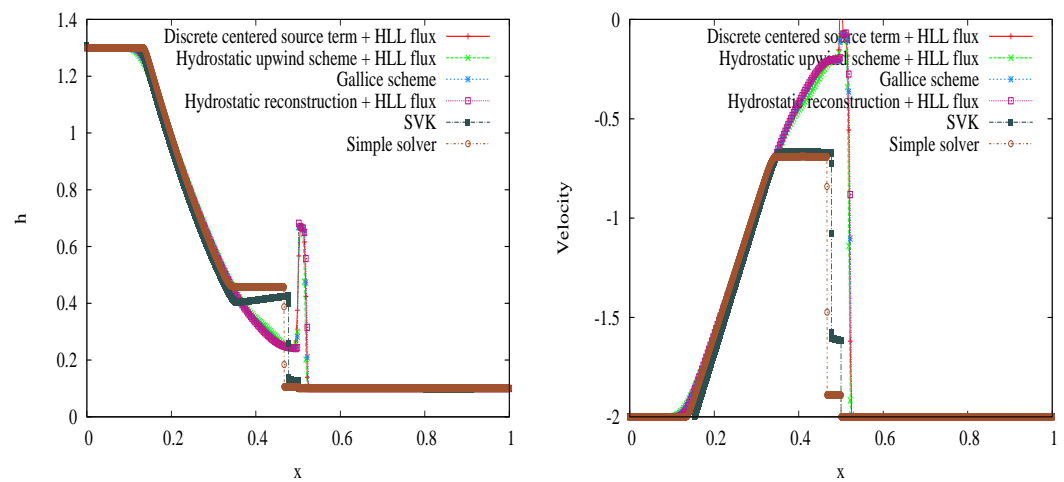

FIG. 3.14. Non-unique solution test case: Comparison of water heights $h$ (left) and velocities $u$ (right) for different schemes.

We present the value of the two entropies (1.4) and (1.6) integrated over the domain as a function of time for different schemes on Fig. 3.15. The idea is to numerically bring out the behaviour of the entropy. It appears that the conservative entropy as well as the non-conservative one, calculated for the proposed scheme always decreases and so satisfies at least numerically the entropic criterion. On the contrary, this observation cannot be applied for the other ones in which a period of increasing entropy takes place. In a quantitative point of view, one notes that the values of the non-conservative entropy is lower than those of the conservative one. In fact, by recalling the two entropy inequalities (1.3) and (1.5), we can show that the derivative value for the conservative entropy has to be lower than zero whereas the same quantity for the non-conservative entropy has a negative upper bound causing that the nonconservative entropy decreases faster than the conservative one.

\section{Conclusion.}

In this paper, we have proposed a simple to implement, positive and well-balanced scheme for the shallow water equations. The scheme proved to be very accurate on the several typical test cases: propagation of perturbation, stationary and nonstationary shock, wet-dry transition on a smooth and non smooth bottom topography. Furthermore, we have shown that it converges towards the right entropy solution in a resonant test case. The very motivation of this work comes from the numerical approximation of the solutions of the Saint-Venant-Exner equations for the problem of sediment bedload transport, to which we would like to adapt the proposed scheme therein. This is the matter of a work currently in progress.

Acknowledgements. The authors would like to thank the reviewer for his thorough reading and his relevant remarks. P. U. would like to thank AMIES and 

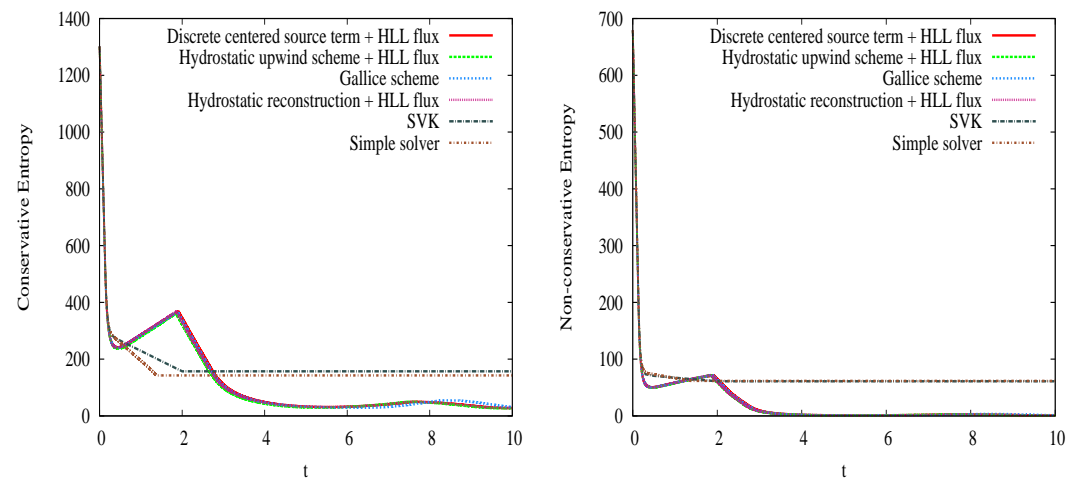

FiG. 3.15. Non-unique solution test case: Comparison of the conservative (left) and nonconservative (right) entropy for different schemes.

EDF for their financial support.

\section{REFERENCES}

[1] N. Andrianov, Performance of numerical methods on the non-unique solution to the Riemann problem for the shallow water equations, Internat. J. Numer. Methods Fluids, 47, 825-831, 2005.

[2] E. Audusse, F. Bouchut, M.-O. Bristeau, R. Klein and B. Perthame, A fast and stable wellbalanced scheme with hydrostatic reconstruction for shallow water flows, SIAM J. Sci. Comp., 25, 2050-2065, 2004.

[3] D. S. Bale, R. J. Leveque, S. Mitran and J. A. Rossmanith, A wave propagation method for conservation laws and balance laws with spatially varying flux functions, SIAM J. Sci. Comput., 24, 2002.

[4] C. Berthon and C. Chalons, A fully well-balanced, positive and entropy-satisfying Godunov-type method for the shallow-water equations, HAL:hal-00956799, version 1, http://hal.archivesouvertes.fr/hal-00956799, 2014.

[5] C. Berthon and F. Foucher, Efficient well-balanced hydrostatic upwind schemes for shallowwater equations, J. Comput. Phys., 231, 4993-5015, 2012.

[6] F. Bouchut, Nonlinear stability of finite volume methods for hyperbolic conservation laws and well-balanced schemes for sources, Frontiers in Mathematics, Birkhäuser Verlag, 2004.

[7] F. Bouchut and T. Morales, A subsonic-well-balanced reconstruction scheme for shallow water flows, SIAM J. Numer. Anal., 48, 1733-1758, 2010.

[8] M. Castro, J. Macias and C. Pares, A Q-scheme for a class of systems of coupled conservation laws with source term, application to a two-layer 1-D shallow water system, M2AN Math. Model. Numer. Anal., 35, 107-127, 2001.

[9] M. J. Castro, A. Pardo, C. Pares and E. F. Toro, On some fast well-balanced first order solvers for nonconservative systems, Math. Comp., 79(271), 1427-1472, 2010.

[10] C. Chalons, F. Coquel, E. Godlewski, P.-A. Raviart, N. Seguin, Godunov-type schemes for hyperbolic systems with parameter dependent source. The case of Euler system with friction, Mathematical Models and Methods in Applied Sciences (M3AS), 20(11), 2010.

[11] A. Chinnayya, A.-Y. Leroux and N. Seguin, A well-balanced numerical scheme for the approximation of the shallow-water equations with topography: the resonance phenomenon, International Journal on Finite Volumes, 1, 1-33, 2004.

[12] G. Gallice, Solveurs simples positifs et entropiques pour les systèmes hyperboliques avec terme source, C. R. Math. Acad. Sci. Paris, 334(8), 713-716, 2002.

[13] G. Gallice, Positive and entropy stable Godunov-type schemes for gas dynamics and MHD equations in Lagrangian or Eulerian coordinates, Num. Math. 94 no.4, 673-713, 2003.

[14] T. Gallouët, J.-M. Hérard and N. Seguin, Some approximate Godunov schemes to compute shallow-water equations with topography, Computers and Fluids, 32, 479-513, 2003.

[15] A. Harten, P.-D. Lax and B. van Leer, On upstream differencing and Godunov-type schemes for hyperbolic conservation laws, SIAM Review, 25(1), 53-61, 1983. 
[16] R. LeVeque, Balancing source terms and flux gradients in high-resolution Godunov methods: The quasi-steady wave-propagation algorithm, J. Comput. Phys., 146, 346-365, 1998.

[17] T. Morales de Luna, M. J. Castro Diaz and C. Pares, Reliability of first order numerical schemes for solving shallow water system over abrupt topography, Applied Mathematics and Computation, 219, 9012-9032, 2013.

[18] B. Perthame and C. Simeoni, A kinetic scheme for the Saint-Venant system with source term, Calcolo, 38, Springer-Verlag, 201-231, 2001. 